\title{
Sharpness evaluation of microscopic detection image for micro parts
}

\author{
Xianghui Zhang, Zhanjiang Yu, Jinkai Xu, and Huadong $\mathrm{Yu}^{*}$ \\ Ministry of Education Key Laboratory for Cross-Scale Micro and Nano Manufacturing, Changchun \\ University of Science and Technology, Changchun, China
}

\begin{abstract}
According to the characteristics of micro parts microscopic detection image, including the image texture is similar, the edge information is too little and the gray distribution Range is limited, based on the basic principles of algorithm, analyzes the traditional sharpness evaluation function. Aiming at the defect that the traditional sharpness evaluation function cannot have both high sensitivity and noise immunity, an algorithm based on local variance information entropy is proposed. The method uses the local variance to weight the self-information of each gray level,on the one hand, it makes up for the lack of spatial information of information entropy and avoids misjudgement of sharpness; on the other hand, it can increase the weights of clear region pixels when they participate in the calculation of information, while reducing the weights of background and noise region pixels, thereby improve the function sensitivity. The experimental results show that compared with the traditional sharpness evaluation function, the local variance information entropy function not only has high sensitivity, but also has better noise immunity and is suitable for actual auto-focusing systems.
\end{abstract}

Keywords: Auto-focusing, Sharpness evaluation, Local variance information entropy, Micro parts, Microscopic image

\section{Introduction}

The emergence of nanotechnology, micro-machine and micro-electromechanical systems has promoted the rapid development of precision machining technology of microparts [1], and then higher requirements have been put forward for the detection of small parts. The detection method based on machine vision has reliability. It has many advantages such as high precision, non-contact measurement, etc. [2]. It is often used to micro-parts surface morphology observation, surface defect detection and geometric size measurement, etc.

In order to realize the automation and intelligent testing process, which requires the machine vision detection system has autofocus function. The key technology of autofocus system based on image processing including image sharpness evaluation and search

\footnotetext{
* Corresponding author: yuhuadong@,cust.edu.cn
} 
algorithm [3], must carry on the autofocus, first of all need to be able to judge whether the image can be obtained as the clear focus of the image, The sharpness evaluation function is used to score the images taken at different positions, so that the sharpness of the images can be obtained quantitatively and then the focusing distance between the lens and the parts can be determined and a clear focus image can be obtained.

Tiny parts of microscopic detection based on machine vision, when meet the high precision, because of the high magnification lens. The depth of field is very small, which leads to the focus position fluctuation range is very small, so by definition get clear image focusing evaluation function accurately and quickly is the foundation of subsequent image processing and analysis, it is the premise to guarantee the overall precision.

Most of the sharpness evaluation functions are used for autofocus technology, so the ideal image sharpness evaluation functions need to be unbiased, unimodal, anti-noise, high sensitivity and small calculation amount [4,5]. At first, this paper analyses the characteristics of small parts microscopic detection images, then aiming at the characteristics of such images, starting from the basic principle of algorithm, the performance of the traditional definition evaluation function are analyzed and compared, for the existence of some defects, this paper proposes a definition evaluation algorithm based on local variance information entropy, the last is verified by experiments.

\section{Definition evaluation function}

The research on image sharpness evaluation function has a long history and many classical algorithms have appeared successively, which can be divided into frequency domain function, gradient function, statistical function and informatics function according to the principle [6].

\subsection{Frequency domain functions}

The principle of this kind of algorithm is that the low-frequency information of the image reflects the contour of the image, while the high-frequency information reflects the edge and details of the image, while the clearly focused image has a clear edge and rich details of the image, so the component of the high-frequency part is larger. The algorithm is mainly represented by Fourier transform and wavelet transform in form. By choosing some form of basis function, the image is transformed from spatial domain to frequency domain, and the sharpness of the image is measured according to the distribution characteristics of the image in the frequency domain. This algorithm has high accuracy, but the computation is too large, so it is not suitable for real-time microscopic detection of tiny parts.

\subsection{Gradient function}

This algorithm uses the characteristics of image space to calculate image sharpness. The principle is that the edge of a clear image is clear and distinct, so that the gray value difference between adjacent pixels is large, which means that the gradient of image gray is large. However, the edge of the blurred image is not clear, the gray value difference between adjacent pixels is small and the gray gradient of the image is small. The more representative ones are Brenner function, Tenengrad function and Laplacian function. The common feature of these algorithms is to use some kind of spatial domain template to carry out spatial filtering of images. The difference is that the gradient calculation method is different, that is, the template is different. This kind of function usually has simple algorithm, small computation and high sensitivity. However, due to the similar texture of 
microscopic images of tiny parts, the lack of edge information, and the slow change of image gradient in the process of focusing and defocusing, the sensitivity of the images is reduced compared with the general target images. Moreover, this kind of function is sensitive to noise [6], especially the Laplacian function of the second-order differential operator.

\subsection{Statistical function}

This kind of algorithm uses image statistics information to calculate image sharpness. At present, this kind of function is few in number, and Variance function is the most commonly used one in practice. Grayscale variance is a useful measure of image contrast [7], and a clear image has a higher contrast and a greater difference in gray level than a fuzzy image [8]. Therefore, the variance function can be used as a sharpness evaluation function and its expression is as follows:

$$
F=\sum_{x} \sum_{y}[f(x, y)-\mu]^{2}
$$

where, $f(\mathrm{x}, \mathrm{y})$ is the gray value at the pixel point of the image, and $\mu$ is the average gray value of a large or small of $\mathrm{M} \times \mathrm{N}$ image. This evaluation function requires little computation and can resist the interference of noise [9], but its disadvantage is that its sensitivity is very low.

\subsection{Informatics functions}

This kind of algorithm believes that a clear image contains more information than a fuzzy one, and the amount of information can be measured by the information entropy of the image. Therefore, the information entropy of the image can be used to measure the clarity of the image, that is, the entropy value of a clearly focused image is higher. The concept of information entropy [10] is derived from Shannon's information theory, and the clarity evaluation function based on information entropy is similar to the definition of entropy in information theory. Its expression is as follows:

$$
H=-\sum_{i=0}^{L-1} p_{i} \log \left(p_{i}\right)
$$

where is the occurrence probability of each gray level, and $\mathrm{L}$ is the total number of image gray levels. The algorithm of the evaluation function is simple and the calculation amount is small, but its sensitivity is low. At the same time, because it only considers the probability information of gray value, but does not reflect the spatial information of the image, the extracted features lose a lot of useful information, which is easy to cause the definition misjudgment. This feature is especially obvious when the texture of microscopic images of tiny parts is similar and the gray distribution of adjacent images is similar in the focus and defocus.

To sum up, for microscopic detection images of small parts, frequency domain function consumes too much time and is not suitable for real-time detection. Therefore, such algorithm is not considered in this paper. The gradient function can improve the sensitivity of the local gray scale change but also increase the influence on the noise, and the sensitivity of the target image is reduced compared with the general target image. Although the statistical function of inverse variance has good anti-noise performance, it lacks the description of local gray information, so its sensitivity is very low. However, informatics 
function has certain application limitations because it loses all spatial information. Aiming at the shortcomings of the traditional definition evaluation function, a definition evaluation algorithm based on local variance information entropy is proposed in this paper. The algorithm has high sensitivity and good anti-noise performance while ensuring unbiased and unimodality.

\section{Clarity evaluation function based on local variance information entropy}

According to Shannon information theory, the self-information contained in each gray level [11] is ,

$$
H_{i}=-\log \left(p_{i}\right) t
$$

The information entropy of the image is the weighted average of the self-information $H_{i}$ of all gray levels, and the weighted value is $\mathrm{p}_{\mathrm{i}}$, the probability of the occurrence of each gray level, namely the information entropy.

$$
H=\sum_{i=0}^{L-1} p_{i} H_{i}=-\sum_{i=0}^{L-1} p_{i} \log \left(p_{i}\right)
$$

Formula (4) assumes that the positions of pixels corresponding to the same gray level are $\left(x_{1}, y_{1}\right),\left(x_{2}, y_{2}\right) \cdots\left(x_{n}, y_{n}\right)$,the local variance corresponding to each gray level is defined as:

$$
\begin{aligned}
\sigma_{i}^{2} & =\sum_{j=1}^{n} \sigma_{\left(i, x_{j}, y_{j}\right)}^{2} \\
& =\sum_{j=1}^{n} \frac{1}{9} \sum_{a=-1}^{1} \sum_{b=-1}^{1}\left[f\left(x_{j}+a, y_{j}+b\right)-\mu\right]^{2}
\end{aligned}
$$

where $\sigma_{\left(i, x_{j}, y_{j}\right)}^{2}$ is the local variance of the $3 \times 3$ neighborhood of the image as $\left(x_{j}, y_{j}\right)$ the center point, $\mu$ is the average gray level of the neighborhood, $f\left(x_{j}+a, y_{j}+b\right)$ is the gray level at the pixel point of the image $\left(x_{j}+a, y_{j}+b\right)$.

Then the local variance information entropy is defined as:

$$
H_{\mathrm{Var}}=-\sum_{i=0}^{L-1} p_{i} \sigma_{i}^{2} \log \left(p_{i}\right)
$$

where, $\log$ refers to the logarithm. Different logarithmic bases only determine the unit of entropy and do not affect the focusing performance. In information theory, it is generally taken as $2 . p_{i}$ is the occurrence probability of each gray level, and $L$ is the total number of image gray levels. Since the gray resolution of the image is usually 8 bits [7], $L=2^{8}=256$.

As mentioned above, the calculation of image information entropy is only based on the statistics of gray histogram and lacks the local features of pixels. Therefore, the local variance of pixels belonging to the same gray level is calculated respectively. The calculated local variance is evaluated after weighting the self-information of each gray level. Compared with the information entropy, the improved function in this paper takes into account not only the probability information of gray level, but also the spatial information 
of the image, so as to avoid the misjudgment of clarity easily caused by the information entropy and reflect the overall image clarity in a more comprehensive way. On the other hand, the same as the principle of variance, the local variance can represent the local clarity of the neighborhood centered on the corresponding position of each gray level. The selfinformation weighting of the gray level can increase the weight of the pixels in the clear region when they participate in the calculation of information, while reducing the weight of the pixels in the background region and the noise region. Therefore, the local variance information entropy function should have high sensitivity and good anti-noise.

\section{Experimental results and analysis}

\subsection{Analysis of actual test results}

In order to verify the effectiveness of local variance information entropy algorithm is proposed in this paper, to avoid possible contingency, A target image based on super depth of field microscope to precision machining after two small parts, respectively, near the focus position collected 21 images have A, B two groups of images, Figure. 1 for the two groups of different from focal degree of images in image sequence. The definition evaluation function mentioned above is used to test the actual image sequence from defocus to focus and then to defocus. After normalization, the definition evaluation curve of each function is shown in Figure. 2. It can be seen from the figure that the evaluation results of the clarity of each function are consistent with the theoretical analysis above. In the evaluation of the 10th image in Figure. 2 (B), the information entropy function caused definition misjudgment, resulting in a small difference in the definition value between the 10th image and the 11th image (the unnormalized definition value of the 10th image is 6.8302, and the 11th image is 6.8328), which makes it difficult to accurately distinguish the focused images. Except for information entropy, other sharpness evaluation functions can accurately find the clear focused image (the 11th picture). The evaluation function curve has only one maximum point, which decreases monotonically on both sides of the maximum point, and all of them meet the requirements of unbiased and unimodality. In terms of sensitivity, when the variance and information entropy function are used to evaluate the microscopic detection images of small parts, they are the same as the general images. The curves on both sides of the maximum value are flat and the sensitivity is very low. When the gradient function is used to evaluate the microscopic detection images of small parts, the sensitivity is reduced compared with the general images. This feature is particularly evident in the Brenner and Tenengrad functions in Figure. 2 (A). Laplacian is a second order differential operator, because of the gradient changes. The response amplitude is stronger than the first order differential operator, so the sensitivity is higher than other gradient functions. However, the definition evaluation curve of the local variance information entropy function basically coincides with the Laplacian function, which has a high sensitivity. Therefore, in general practical focusing, the performance of the proposed local variance information entropy function and Laplacian function is superior to other sharpness evaluation functions for microscopic detection images of small parts.

\subsection{Experimental simulation of anti-noise performance}

When CCD or CMOS camera collects images, illumination level and sensor temperature are the main factors that affect the amount of noise in the image [7]. The image sequence used in the above experiment is collected in practice and contains noises introduced in the process of image acquisition and transmission. Therefore, the definition evaluation curve of 
each function actually contains the response to noise. At the same time, the microscopic detection environment of precision machined tiny parts is generally light stable, and most industrial cameras have automatic exposure and automatic filtering functions. Therefore, under general detection conditions, the curve in Figure. 2 can reflect the performance of each sharpness evaluation function.

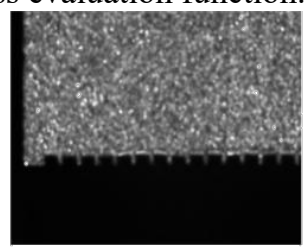

(a) Depth from defocus (A 1st)

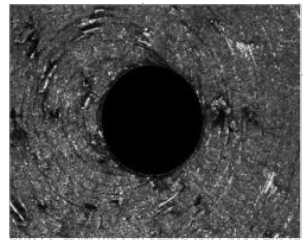

(d) Positive defocus (B 11th)

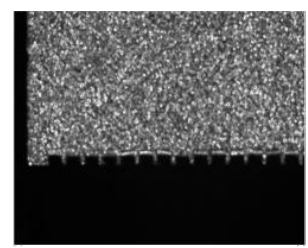

(b) Slight from defocus (A 8 th)

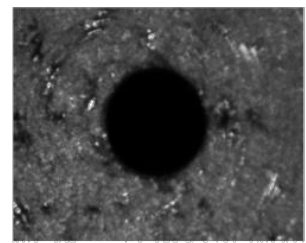

(e) Slight from defocus (B 16th)

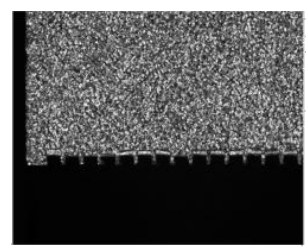

(c) Focus (A 11th)

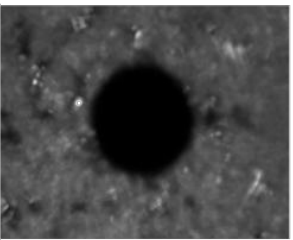

(f) Depth from defocus (B 21th)

Fig. 1. Images with different degrees of defocus.
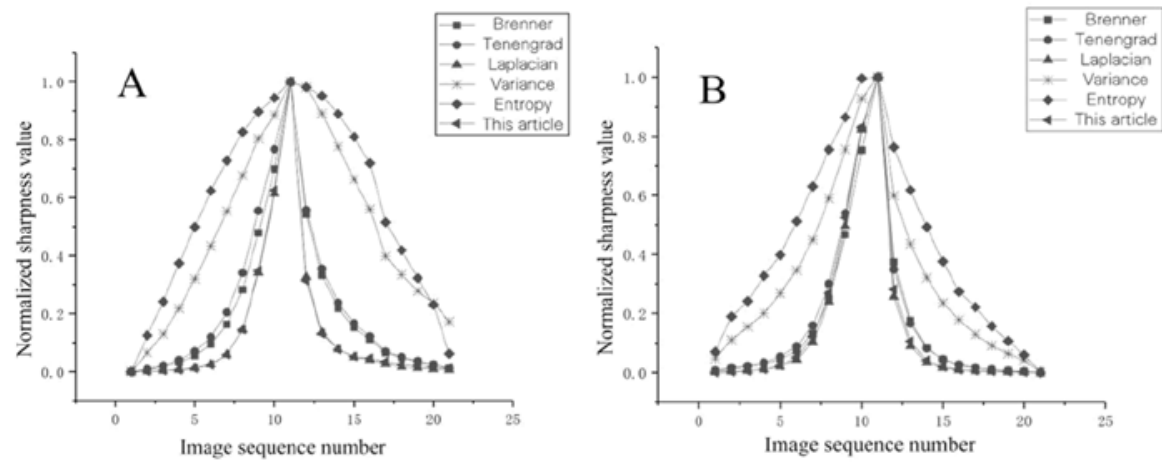

Fig. 2. Clarity evaluation curve of each function.

However, considering that in extreme detection environment, Gaussian noise may be generated when the temperature of the camera's sensor is too high or the illumination intensity is low or uneven for a long time [9], this paper adds Gaussian noise (mean value is 0 , variance is 0.01 ) to the actual shot image sequence to simulate the noise that may be introduced in extreme detection environment. Figure. 3 is the definition evaluation curve of each function after adding noise. It is not difficult to see from the figure that, in extreme detection environment, the second-order differential is more radical than the first-order differential in response to drastic changes, so the Laplacian function is particularly sensitive to noise, and the function curve shows local extreme value, and even fails in group B images with poor anti-noise performance. While the local variance information entropy function proposed in this paper, although the curve of the function is not smooth enough at far-focus, the function can still accurately find the clear focused image and maintain a high sensitivity, which indicates that the algorithm proposed in this paper has a good anti-noise performance. 

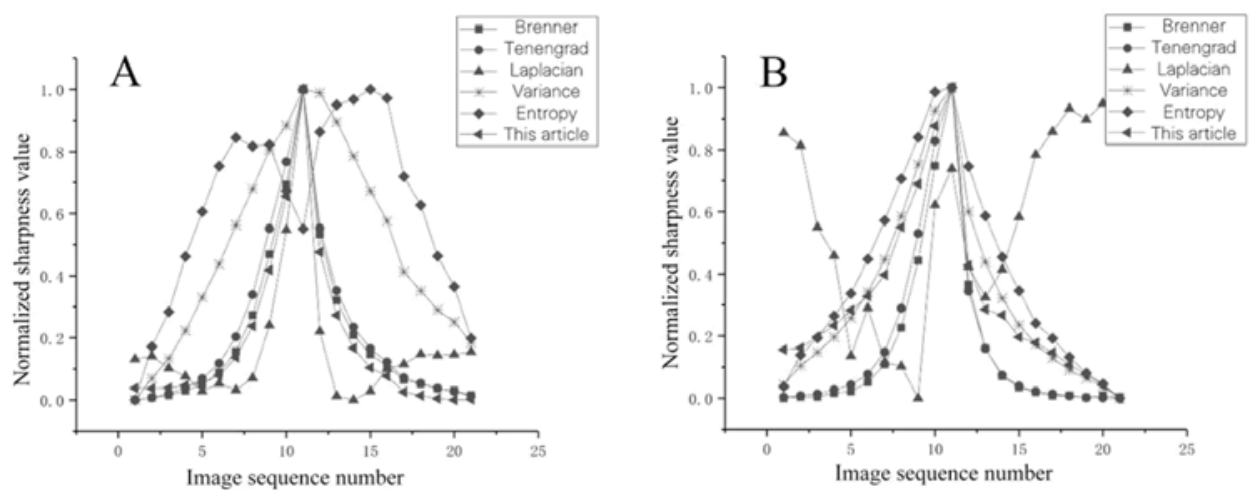

Fig. 3. Clarity evaluation curve of each function after.

\section{Conclusion}

For microscopic inspection images of small parts, this paper proposes a definition evaluation algorithm based on local variance information entropy, aiming at the problems that the gradient function has a decreased sensitivity and is sensitive to noise, the variance function can resist noise interference but has a low sensitivity, and the information entropy function loses spatial information and is prone to misjudgment of clarity. Through the actual test and the simulation of anti-noise performance, it can be seen that the performance of this algorithm is better than other algorithms in the general actual detection, and the sensitivity is very high. Even when noise is introduced into the extreme detection environment, the algorithm can still find the clearly focused image accurately with high sensitivity and good anti-noise performance.

This work is supported by Jilin Province Scientific and Technological Development Program (No.Z20190101005JH). Young Scientist Fund of Changchun University of Science and Technology (No.XQNJJ-2018-09).

\section{References}

1. Z. Yuan, X. Wang, "Precision and Ultraprecision Machining Technology. 3rd Edition," China Machine Press, 2016.

2. Q. Hou. "Investigation on the technology of tool wear detection based on machine vision," Shandong University, 2018.

3. Y. You, T. Liu, J. Liu, "Survey of the auto-focus methods based on image processing,". Laser \& Infrared, vol.43, pp.132-136,2013.

4. Wang Jian. "Study on technology of auto-focus based on image processing," Graduate School of Chinese Academy of Sciences (Institute of Optics and Electronics), 2013.Another reference

5. Y, Zhai, D. Zhou, Y. Liu, S. Liu. K. Peng, "Design of evaluation index for autofocusing function and optimal function selection,". Acta optica Sinica,vol.31, pp.234$244,2011$.

6. Y. Hong, G. Ren, J. Sun. “Analysis and improvement on sharpness evaluation function of defocused image," Optics and Precision Engineering, vol.22, pp.3401-3408,2011.

7. Gonzalez R C, Woods R E. Digital Image Processing (3rd Ed.). 2007. 
8. Q. Li, H. Feng, Z. Xu, M. Bian, S. Shen, R. Dai, "Study on the sharpness evaluation function of digital image,” Acta PHOTONICA Sinica, vol.31, pp.736-738,2002.

9. X. Zheng, L. Ai, K. Liu, B. Su. "Auto-Focusing function for microscopic images based on global and local gray-scale variation," Laser \& Optoelectronics Progress, vol.54, pp.246-253,2017.

10. Shannon C E. A mathematical theory of communication. Bell Labs Technical Journal, vol.27, pp.379-423,1948.

11. X. Cao, Z, Zhang. "Information theory and coding (2nd Edition)," Beijing: Tsinghua University Press, 2009. 